\title{
Synthesis of ZnSe@ZnS core-shell quantum dots (QDs) for bioimaging applications
}

Yang He

Follow this and additional works at: https://researchrepository.wvu.edu/etd

\section{Recommended Citation}

He, Yang, "Synthesis of ZnSe@ZnS core-shell quantum dots (QDs) for bioimaging applications" (2017). Graduate Theses, Dissertations, and Problem Reports. 3999.

https://researchrepository.wvu.edu/etd/3999

This Problem/Project Report is protected by copyright and/or related rights. It has been brought to you by the The Research Repository @WVU with permission from the rights-holder(s). You are free to use this Problem/Project Report in any way that is permitted by the copyright and related rights legislation that applies to your use. For other uses you must obtain permission from the rights-holder(s) directly, unless additional rights are indicated by a Creative Commons license in the record and/ or on the work itself. This Problem/Project Report has been accepted for inclusion in WVU Graduate Theses, Dissertations, and Problem Reports collection by an authorized administrator of The Research Repository @ WVU. For more information, please contact researchrepository@mail.wvu.edu. 


\title{
Synthesis of ZnSe@ZnS Core-Shell Quantum Dots (QDs) For Bioimaging Applications
}

\author{
Yang He \\ Problem Report submitted \\ to the Benjamin M. Statler College of Engineering and Mineral Resources \\ at West Virginia University \\ in partial fulfilment of the requirement for the degree of \\ Master of Science in Material Science and Engineering \\ Edward Sabolsky, PhD, Chair \\ Konstantinos A. Sierros, PhD \\ Terence Musho, $\mathrm{PhD}$ \\ Department of Mechanical and Aerospace Engineering \\ Morgantown, West Virginia \\ 2017
}

Keywords: ZnSe@ZnS, Core-shell, Quantum Dots, Bioimaging

Copyright 2017 Yang He 


\begin{abstract}
Synthesis of ZnSe@ZnS Core-Shell Quantum Dots (QDs) For Bioimaging Applications

Yang He
\end{abstract}

To date, malignancies have been categorized as one of the leading life-threatening diseases worldwide, mainly because of lack of imaging devices for detection of cancers at its early stage of development. In this case, the emergence of novel strategies which can be effectively used in early stage cancer detection and therapy continue to become an inevitable need and indispensable scope of and cancer field researchers, for which implementation of new technologies are required to advance the early detection and management of cancers. The biomedical imaging of the organs in the living system offers a viable approach to label and image the cells. Among the different bioimaging techniques such as X-ray, Commuted Tomography, Emission Tomography and Magnetic Resonance Imaging that have been developed, Quantum-Dots based technique has acquired increasing attention because of its unique optical and electrical properties and good biocompatibility. In this report the synthesis of ZnSe@ZnS core-shell Quantum Dots, which is cadmium-free and non-toxic to the biological cells, is proposed. These core-shell Quantum Dots will be applicable to biomedical imaging and diagnostics operating on living biological cells. 


\section{ACKNOWLEDGEMENTS}

First of all I would like to express my sincere gratitude to my Report Advisor Dr. Edward Sabolsky for his support and guidance. I deeply appreciate his inputs for this report. I'm also grateful to Dr. Terence Musho and Dr. Konstantinos Sierros for accepting to be a member of my report committee. I thank them for their insightful comments and encouragement.

I would also like to thank my friends for their support and great help. I thank them for listening to me whenever I needed it and standing by my side all the time.

Finally, I must express my very profound gratitude to my parents and to my husband for providing me with unfailing support and continuous encouragement throughout my years of study and through the process of writing this thesis. This accomplishment would not have been possible without them. Thank you. 


\section{TABLE OF CONTENTS}

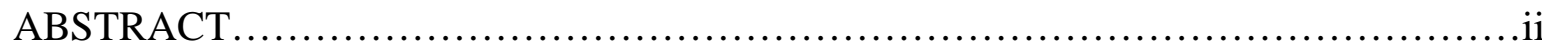

ACKNOWLEDGEMENTS............................................................ii

TABLE OF CONTENTS.....................................................................

LIST OF TABLES ..................................................................

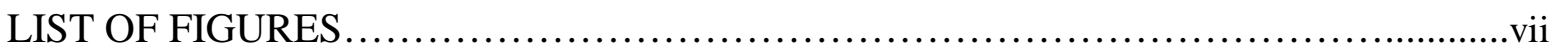

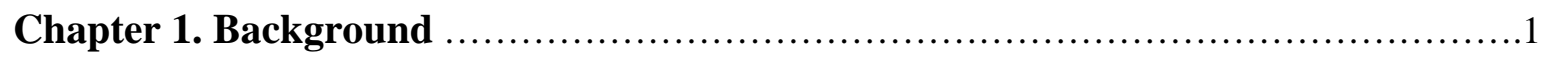

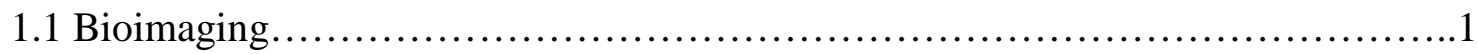

1.2 QDs Technique for Bioimaging.............................................. 1.

1.2.1 Quantum Dots Basic Principles.....................................1

1.2.2 QDs Applications in Bioimaging......................................4

1.2.3 Advantages of QDs for Bioimaging....................................

1.3 Surfactants on QDs for Bioconjugation..........................................

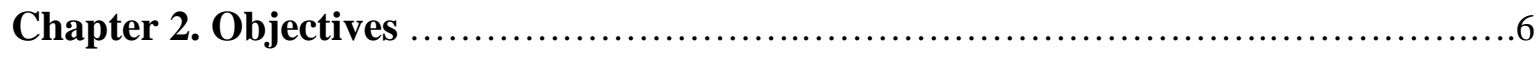

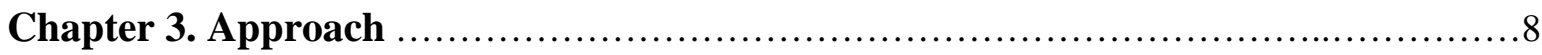

3.1 Organometallic Method.....................................................................................

3.2 Aqueous Solution Based Approach..............................................

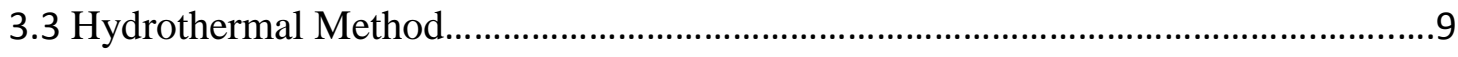

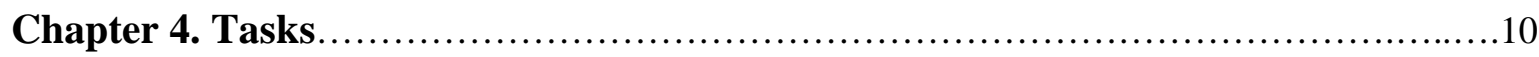

4.1 Task 1. Synthesis and Characterization of ZnSe Core QDs................................10

4.1.1 Synthesis of ZnSe Core QDs................................................10

4.1.2 Characterization of ZnSe Core QDs. ........................................11

4.2 Task 2. Synthesis and Characterization of ZnSe/ZnS Core-shell QDs......................12

4.2.1 Synthesis of ZnSe/ZnS Core-Shell QDs........................................12

4.2.2 Characterization of ZnSe/ZnS Core-Shell QDs. .............................13

4.3 Task 3. Conjugation of ZnSe@ZnS Core-Shell QDs with U251............................14

4.3.1 Conjugate TGA-Caped ZnSe@ZnS Core-Shell QDs with RGD...................14 
4.3.2 Culture U251 Cell ..................................................... 14

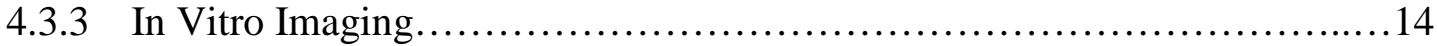

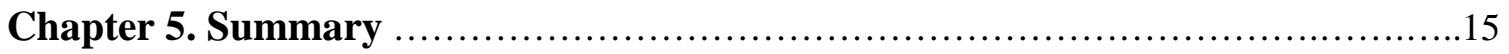




\section{LIST OF TABLES}

Table 1 Desired properties of the synthesized ZnSe@ZnS core-shell QDs

Table 2 Tentative List of Variables for ZnSe Core QDs Synthesis

Table 3 Tentative List of Variables for ZnSe/ZnS Core-shell QDs Synthesis 


\section{LIST OF FIGURES}

Figure 1. Schematic of bulk semiconductor band structure and emission. ${ }^{6}$

Figure 2. Splitting of energy levels in quantum dots due to the quantum confinement effect. ${ }^{7}$

Figure 3. Schematic of quantum dots size and emission wavelength. ${ }^{8}$

Figure 4. Schematic of ligand exchange of hydrophobic TOPO by hydrophilic MAA. ${ }^{13}$ 


\section{Chapter 1. Background}

\subsection{Bioimaging}

Nowadays, malignancies are becoming one type of the leading life-threatening diseases as generally there is no obvious early-stage symptoms for the patients and there is a lack of imaging devices for effective early-stage detection of cancers which hinders the early-stage control and therapy of the tumors. Thus the research into novel imaging techniques are of great interests and continue to be an inevitable need. ${ }^{1}$

Bioimaging is a relatively recent development in the medical field that makes use of digital technology and the bioimaging techniques offer a viable approach to label and image cells in living systems in real time. ${ }^{2}$ Bioimaging is a term covers a complex chain of acquiring, processing and visualizing structural or functional images of living objects or systems and it includes both the extraction and processing of image-related information. ${ }^{3}$

There are different kinds of bioimaging techniques, such as the X-ray, Ultrasound, CT (Commuted Tomography), ET (Emission Tomography), MRI (Magnetic Resonance Imaging), Quantum-Dots (QDs) techniques. ${ }^{4}$

\subsection{QDs technique for bioimaging}

Among these different bioimaging techniques, QDs is a promising technique because of its good optical properties and has attracted increasing attention among researchers in this field.

\subsubsection{Quantum Dots Basic Principles}

Briefly, a Quantum Dot (QD) is a semiconductor material structure with very small size. This size should be small enough to confine its excitons in all three spatial dimensions. As the structure of the QDs are between that of a bulk semiconductor and a molecule, the electronic properties would thus be intermediate between those of bulk semiconductors and of discrete molecules. Since first discovered by Alexey Ekimov in $1981^{5}$, Quantum Dots has become a very hot topic for these three decades. Generally the size of the Quantum-Dots are 2-10nm in diameter. 
In a bulk semiconductor material, the distribution of states for electron density are separated into two bands, the conduction band (CB), and the valence band (VB). And the energy bandgap between these two bands are generally less than $4 \mathrm{eV}$. When light is incident on the semiconductor and the photon energy of the solar light is greater than the bandgap of this semiconductor, the photon can be absorbed by the semiconductor. This photon energy can be utilized to excite electrons from the valence band up to the conduction band. Later on, these electrons in the CB could jump back to the VB and release the energy in the form of emitting light, as is shown in Figure 1.

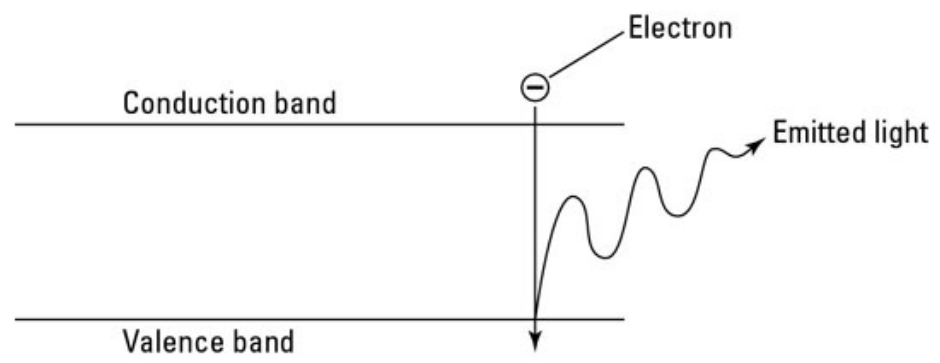

Figure 1 Schematic of bulk semiconductor band structure and emission. ${ }^{6}$

Whereas for the QDs, the conduction band and valance band become discrete and quantized energy levels, as is shown in Figure 2. ${ }^{7}$ The difference in energy between the highest level of valence band and the lowest level of conduction band is related to the size of the QDs. As the size of the QDs decreases, this difference would increase. Based on this, photons with higher energy need to be absorbed by the QDs upon irradiation to excite the dot, and concurrently, more energy is released when the electron from excited energy state jumps back to the ground state, therefore the color of the emitted light would shift from the red end to the blue end. As a result of this phenomenon, quantum dots can emit different color of light from the same material simply by changing the dot size, which is shown in Figure 3. ${ }^{8}$ 


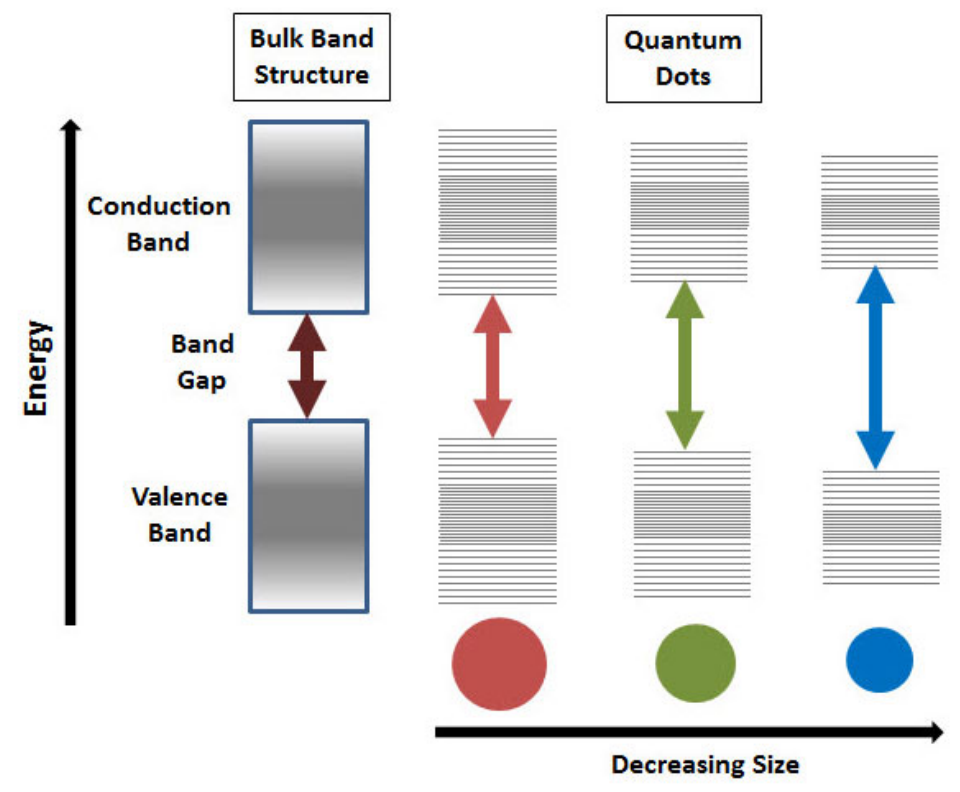

Figure 2. Splitting of energy levels in quantum dots due to the quantum confinement effect. ${ }^{7}$

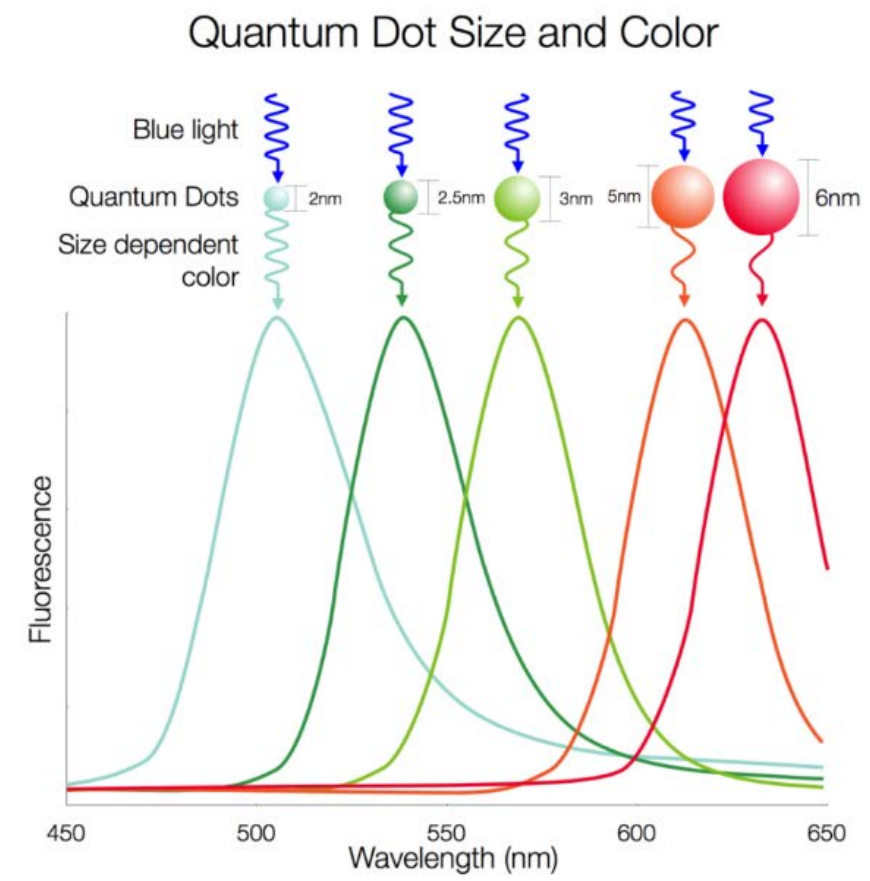

Figure 3 Schematic of quantum dots size and emission wavelength. ${ }^{8}$ 


\subsubsection{QDs Applications in Bioimaging}

When the Quantum dots (QDs) are encapsulated with amphiphilic polymer surfactants and then bioconjugated with different molecules or cells in living system, these QDs can be utilized as probes for the detection and imaging of this living systems.

QDs has been reported as a viable probe for long-term labeling of human Mesenchymal Stem Cells (hMSC) ${ }^{9}$ In this study, the quantum dots remained incorporated in the hMSCs for 22 days, which suggests that bioconjugated quantum dots have long-term labeling capability for stem cells.

Quantum dots can target prostate cancer and this bioconjugated probe of QD-PSMA (Prostate Specific Membrane Antigen) shows active emission in the presence of C4-2 prostate cancer cells. ${ }^{10}$ Based on this report, the QDs can also be used to passively target tumors since leaky tumor vasculatures retain more quantum dots than surrounding healthy tissue. .

\subsubsection{Advantages of QDs for Bioimaging}

For the optical properties, the conventional fluorophores generally suffer from photobleaching and is not appropriate for long-term utilization. In comparison, the QDs is relative stable and thus shows more stable absorption and emission properties, which is necessary for long-term imaging of targeted cells. ${ }^{4}$

\subsection{Surfactants on QDs for Bioconjugation}

During the process of synthesizing QDs, surfactants are needed to encapsulate the QDs nuclei and then control the size and shape of the QDs in the later growth process under heating. And more importantly, after the synthesis of QDs, the surfactant needs to keep these QDs dispersed well in the solvent and protect them from aggregation. ${ }^{11}$

In order to be utilized for bioimaging application, the synthesized QDs must be made watersoluble. In general, these encapsulated QDs should have stable photo-physical properties in light absorption and emission and should retain relatively small particle size. The surfactants on quantum dots surface should be tailored to target specific cancer lines by providing reactive groups for subsequent conjugation to biomolecules and cells. ${ }^{12}$ 
There are several different approaches to produce water-soluble QDs for bioconjugation. First, we can synthesize the QDs in organic phase and cap the surface by surfactants with hydrophobic groups, and then replace these hydrophobic surface groups with hydrophilic ones by means of ligand exchange. One of the most common process is the substitution of the native TOPO coating with bifunctional ligands, which present both a surface-anchoring group (e.g. thiol) and a hydrophilic end group (e.g. carboxyl or hydroxyl). Examples include utilization of negativelycharged carboxy-terminated thiols, such as mercaptoacetic (MAA) (shown in Figure 4) and mercaptopropionic (MPA) acids. ${ }^{13}$

MAA-QD

TOPO-QD

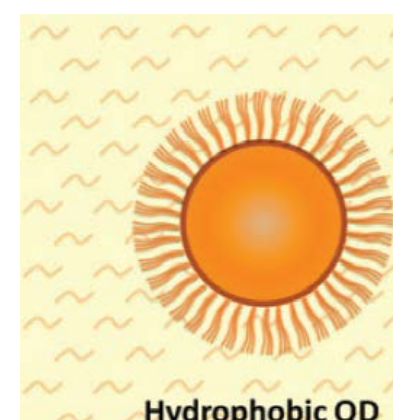

Ligand-exchange

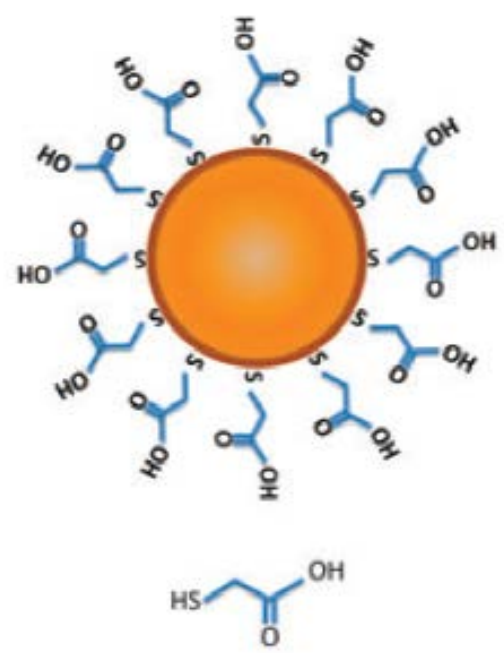

Hydrophilic

Figure 4. Schematic of ligand exchange of hydrophobic TOPO by hydrophilic MAA. ${ }^{13}$

Another route for producing water-soluble QDs is to directly utilize hydrophilic surfactant such as MAA and MPA to decorate the QDs surface during the synthesis process. In this case, no extra ligand exchange process is needed after the synthesis and the QDs can be readily dispersed well in water, which is more convenient for bioimaging application. 


\section{Chapter 2. Objectives}

The primary central nervous system tumors are the third leading cause of cancer-related deaths in the less than 60 -year-old population although this category of tumors represent only $2 \%$ of all new cases of cancer in the U.S. ${ }^{14}$ Glioma is one of the most common primary brain tumors, accounting for about $30-40 \%$ of all intracranial tumors. ${ }^{15}$ Current imaging modalities are unable to take full advantage of the unique nature of protein expression in the tumor cell for early diagnosis. The gliomas show invasive growth with single-cell invasion or penetration of small cell clusters in the surrounding tissue. ${ }^{16}$ Because of this, the differentiation of brain and tumor tissues remains extremely difficult for the surgeon, and incomplete resections are quite common. Thus, development of new techniques for imaging these glioma tumors at cell level are urgently required.

One potential solution to this problem is to use QDs technique, which has been investigated by Liu and coworkers. ${ }^{17}$ But as the QDs materials they used was CdTe, which contains the highly cytotoxic element of $\mathrm{Cd}$, the development of Cd-free QDs as highly efficient and nontoxic bioimaging agent for targeting and live visualizing of glioma cancer cells are of great interests. Among different QDs materials, ZnSe@ZnS core-shell QDs is a good candidate. It has been found that ZnSe quantum dots with the bulk bandgap of $2.7 \mathrm{eV}$ is a promising candidate for $\mathrm{UV}$ emission applications and it has much lower cytotoxicity compared to Cd-contained QDs such as CdS and CdTe. ${ }^{18}$

Specifically, the solution proposed in this problem report is to synthesize ZnSe@ZnS core-shell QDs based on the method demonstrated by Mir. ${ }^{18}$ But both the core size and the shell thickness need to be tuned to achieve sharper emission peak for photoluminescence, which will be realized by modifying the reaction parameters. More importantly, these QDs will conjugate with human glioma cells (U251) to realize the bioimaging of these glioma cells.

Based on the discussion above, the first objective of this report is to synthesize $\mathrm{ZnSe} / \mathrm{ZnS}$ coreshell QDs with lower energy and time consumption. The size of the ZnSe core will be tuned to be $5 \mathrm{~nm}$. And the shell thickness will be tuned to be $3 \mathrm{~nm}$ and $5 \mathrm{~nm}$. 
After the successful synthesis process, the second objective would be to conjugate these core-shell QDs with human glioma cells. And the in vitro bioimaging of these cells will be conducted to test the effectiveness of the QDs technique.

To achieve these objectives of this problem report, the desired properties of the ZnSe@ZnS coreshell QDs synthesized by the modified process in this problem report is shown in Table 1 below.

\begin{tabular}{|c|c|c|}
\hline \multirow{5}{*}{ ZnSe core QDs } & Size & $5 \mathrm{~nm}$ in diameter, uniform size \\
\hline & Shape & Nanospheres, dispersed well in water \\
\hline & Crystal structure & Same as crystallite Zinc Blende structure, sharp peak \\
\hline & UV-vis absorption & High absorption at $\lambda<430-460 \mathrm{~nm}$ \\
\hline & Photoluminescence & Sharp emission peak with high intensity \\
\hline \multirow{6}{*}{$\begin{array}{l}\text { ZnSe@ZnS } \\
\text { core-shell QDs }\end{array}$} & ZnS Shell thickness & $3 \mathrm{~nm}$ and $5 \mathrm{~nm}$, complete and uniform \\
\hline & \multirow{3}{*}{ Crystal structure } & No peak position shift for ZnSe core; \\
\hline & & Sharp peak; \\
\hline & & Increased intensity for core-shell than that of core-only \\
\hline & UV-vis absorption & Not significantly extended from spectrum of core-only \\
\hline & Photoluminescence & Much higher intensity than that of the core-only \\
\hline
\end{tabular}




\section{Chapter 3. Approach}

There are different synthesis approaches for QDs and they are briefly discussed here below.

\subsection{Organometallic Method.}

Synthesis of QDs by organometallic method is conducted in nonpolar organic solvents, and it is an efficient method to make monodisperse QDs. A good example is the synthesis of CdSe nanocrystals covered by tri-octylphosphine oxide (TOPO) ligand. In a typical process, Dimethylcadmium $\left(\mathrm{Cd}\left(\mathrm{CH}_{3}\right)_{2}\right)$ and Bis(trimethylsilyl)selenium ((TMS) $\left.)_{2} \mathrm{Se}\right)$ is used as organometallic precursors. Under heating between $250^{\circ} \mathrm{C}$ to $300{ }^{\circ} \mathrm{C}$, monodispersed CdSe was obtained. ${ }^{19}$ The growth rate of the QDs is relative slow as the surface of the QDs are tightly bonded with ligands.

This method can produce various types of QDs with high quality. More specifically, the size distribution is uniform and the size can be easily tuned by changing synthesis parameters such as reaction temperature and time. What's more, QDs synthesized by this method could have high quantum yields. Organometallic method is currently used as the most important method to synthesize various QDs.

\subsection{Aqueous Solution Based Method.}

Another important method for QDs fabrication is aqueous solution based method, which was firstly reported by Rajh and coworkers. ${ }^{20}$ In organometallic method, the QDs are capped with hydrophobic ligands such as TOP (tri-octylphosphine) and TOPO, which cannot be dispersed in water. Thus if the QDs need to be used in aqueous environment, ligand-exchange is usually needed to change the surface of the QDs to be hydrophilic. Whereas using aqueous solution based method can skip this step and directly synthesize QDs capped with hydrophilic ligands, just like the thiolcapped CdTe QDs produced in Rajh work. ${ }^{20}$ For the synthesis of CdTe QDs, hydrophilic ligands such as 3-mercaptopropionic acid (3-MPA) and glutathione (GSH) can be utilized to successfully obtain the product in aqueous medium. 
The aqueous solution based methods for QDs synthesis have attracted great interests among researchers in this field since Rahj report because this method is a relative environment-friendly synthesis procedure and has relatively low cost compared to organometallic method. As the QDs synthesized from this method are already capped by the hydrophilic ligands, these QDs can be directly applied in biological researches without ligand exchange procedure.

As to the disadvantage of the aqueous solution based method, the QDs synthesized in this method show low quantum yields with broad full width of half-maximum (FWHM). This is related to the wide size distribution and poor stability in aqueous solution.

\subsection{Hydrothermal Method}

The third type of method utilized the hydrothermal method. This method could help to reduce QDs surface defects that are generated during the growth process, it can also synthesize QDs with relative narrow size distribution and high quantum yields than that of the aqueous solution based method. In a hydrothermal method, the reaction regents are loaded in a sealed container and then heated up to supercritical temperature. High pressure produced by the high temperature would help to efficiently reduce the reaction time and surface defects of QDs. ${ }^{21}$ Under high reaction temperature, the nucleation process and crystal growth can be separated, and this helps to produce QDs with narrow size distribution. ${ }^{22}$

Among these three types of synthesis methods, the Aqueous Solution Based Approach would be utilized in this report in an energy and time-saving procedure. 


\section{Chapter 4. Tasks}

This report is designed with three technical tasks, aimed at facilitating the synthesis of energysaving and time-saving ZnSe/ZnS core-shell QDs and utilizing these core-shell QDs in the imaging of glioma cell lines. The individual tasks are described below:

\subsection{Task 1. Synthesis and Characterization of ZnSe Core QDs.}

In this task, the ZnSe core nanoparticles with $5 \mathrm{~nm}$ in diameter will be synthesized and further be characterized.

\subsubsection{Synthesis of ZnSe Core QDs.}

The chemicals of Zinc acetate, selenium powder, $\mathrm{NaOH}, \mathrm{NaBH}_{4}$ and thioglycolic acid (TGA) will be utilized without any further purification. ZnSe core QDs will be prepared through an aqueous route in a reaction flask at room temperature instead of in a heated auto-clave. First, precursor solution A, which is a fresh selenium precursor of NaHSe solution will be prepared by reacting selenium powder with $\mathrm{NaBH}_{4}$. Then precursor solution $\mathrm{B}$, an aqueous mixture of Zinc acetate and TGA, will be prepared with $\mathrm{pH}$ adjusted to $11 \sim 12$ by $\mathrm{NaOH}$. The solution $\mathrm{A}$ will then be added into solution $\mathrm{B}$ and the molar ratio of the Zn: TGA: NaHSe will be adjusted. The reaction mixture will then be heated to a specific temperature for a time duration and then immediately cool down to room temperature. A minimum of eight synthesis experiments will be conducted and a tentative list of synthesis variables is shown in Table 2.

\begin{tabular}{|c|c|c|c|}
\hline \multicolumn{4}{|c|}{ Table 2. Tentative List of Variables for ZnSe Core QDs Synthesis } \\
\hline Experiment & Zn: TGA:NaHSe molar ratio & Temperature & Time \\
\hline 1 & $2.5: 3: 1$ & $80^{\circ} \mathrm{C}$ & $1 \mathrm{~h}$ \\
\hline 2 & $2.5: 3: 1$ & $80^{\circ} \mathrm{C}$ & $1.5 \mathrm{~h}$ \\
\hline 3 & $2.5: 3: 1$ & $90{ }^{\circ} \mathrm{C}$ & $1 \mathrm{~h}$ \\
\hline 4 & $2.5: 3: 1$ & $90{ }^{\circ} \mathrm{C}$ & $1.5 \mathrm{~h}$ \\
\hline 5 & $2.5: 4: 1$ & $80^{\circ} \mathrm{C}$ & $1 \mathrm{~h}$ \\
\hline 6 & $2.5: 4: 1$ & $80^{\circ} \mathrm{C}$ & $1.5 \mathrm{~h}$ \\
\hline 7 & $2.5: 4: 1$ & $90^{\circ} \mathrm{C}$ & $1 \mathrm{~h}$ \\
\hline 8 & $2.5: 4: 1$ & $90^{\circ} \mathrm{C}$ & $1.5 \mathrm{~h}$ \\
\hline
\end{tabular}




\subsubsection{Characterization of ZnSe Core QDs.}

1. Size and shape

The size and shape of the synthesized nanocrystals will be firstly determined due to the inherent effects of morphology and size on the optical properties of nanocrystals (NCs). High-resolution transmission electron microscopy (HR-TEM) will be used to image and characterize these nanomaterials.

Well dispersed nanospheres with size of $5 \mathrm{~nm}$ in diameter with uniform size distribution will be produced as a good product. Structures with aggregation of the nanoparticles could not be utilized for the next bioimaging application because it could not have distinct emission peak and the size would be too large for the future in vivo imaging of cells.

\section{Crystallite structure and size}

Powder x-ray diffraction (XRD) would be utilized to determine the crystallite structure of these synthesized structure.

To prepare for the sample, the as synthesized structures need to be cleaned by water and then suspended in water again. Then we will drop cast this dispersion of structure onto the Si substrate for analysis.

The crystallite facet of the structure would be determined by comparing its peak positions with that of the standard data. And the size will then be determined from the XRD spectra using DebyeScherer relation which utilizes the peak position and peak full width half maximum (FWHM) as input parameters.

As a good result, these structures will have the same structure as the crystallite Zinc Blende, which has sharp peak for (111), (220) and (311) facets. If the peak width is broad with high background, then the structure is not with good crystallinity and is probably with relative large amounts of defects inside the crystal. 


\section{Photophysical properties}

Then photophysical properties of these NCs will be determined by UV-vis spectrometer and laserscanning confocal microscope for absorption properties and Photoluminescence, respectively.

\section{(1) Absorption- UV-vis Spectrometer}

The light absorption spectrum of these structures will be obtained using the UV-vis Spectrometer. The desired structure will have high absorption intensity at wavelength shorter than 430-460nm.

\section{(2) Photoluminescence- Laser-Scanning Confocal Microscope}

The light emission property of the structures will be determined under UV light excitation. As a good result, a sharp emission peak would be prominent in the photoluminescence spectrum with high intensity.

\subsection{Task 2. Synthesis and Characterization of ZnSe/ZnS Core-shell QDs.}

Based on the results of Task 1, ZnSe/ZnS core-shell QDs will be synthesized through experiment parameters adjustment and characterized by different technique.

\subsubsection{Synthesis of ZnSe/ZnS Core-Shell QDs.}

For the synthesis of ZnSe/ZnS core-shell structures, this report will make precursor solution for the ZnS shell and use $20 \mathrm{ml}$ of aqueous ZnSe dispersion produced from task 1 to accomplish the coating of ZnS shell onto ZnSe core.

To prepare for the ZnS shell precursor solution, the chemical of thiourea will be utilized without any further purification. Certain amount of $\mathrm{Zn}(\mathrm{OAc})_{2}$, TGA, and thiourea will be mixed in water. And $10 \mathrm{ml}$ of the mixture solution will be loaded into a three-neck reaction flask. After that, $20 \mathrm{ml}$ of the ZnSe core QDs dispersion produced in task 1 containing about $0.08 \mathrm{~g}$ of ZnSe will be loaded into a three-neck reaction flask. The $\mathrm{pH}$ of the reaction mixture will be adjusted to 10.5 by $\mathrm{NaOH}$ solution. The reaction mixture will then be heated up to a certain temperature for a specific time period. A minimum of eight synthesis experiments will be conducted and a tentative list of synthesis variables is shown in Table 3. 


\begin{tabular}{|c|c|c|c|}
\hline \multicolumn{4}{|c|}{ Table 3. Tentative List of Variables for ZnSe/ZnS Core-shell QDs Synthesis } \\
\hline Experiment & Zn: TGA: thiourea molar ratio & $\begin{array}{c}\text { Mixture heating up } \\
\text { Temperature }\end{array}$ & $\begin{array}{c}\text { Mixture heating up } \\
\text { Time }\end{array}$ \\
\hline 1 & $1: 1.5: 1$ & $80^{\circ} \mathrm{C}$ & $2 \mathrm{~h}$ \\
\hline 2 & $1: 1.5: 1$ & $80^{\circ} \mathrm{C}$ & $3 \mathrm{~h}$ \\
\hline 3 & $1: 1.5: 1$ & $80{ }^{\circ} \mathrm{C}$ & $4 \mathrm{~h}$ \\
\hline 4 & $1: 1.5: 1$ & $90{ }^{\circ} \mathrm{C}$ & $2 \mathrm{~h}$ \\
\hline 5 & $1: 1.5: 1$ & $90{ }^{\circ} \mathrm{C}$ & $3 \mathrm{~h}$ \\
\hline 6 & $1: 1.5: 1$ & $90^{\circ} \mathrm{C}$ & $4 \mathrm{~h}$ \\
\hline 7 & $1: 2: 1$ & $80^{\circ} \mathrm{C}$ & $2 \mathrm{~h}$ \\
\hline 8 & $1: 2: 1$ & $80^{\circ} \mathrm{C}$ & $3 \mathrm{~h}$ \\
\hline 9 & $1: 2: 1$ & $80^{\circ} \mathrm{C}$ & $4 \mathrm{~h}$ \\
\hline 10 & $1: 2: 1$ & $90^{\circ} \mathrm{C}$ & $2 \mathrm{~h}$ \\
\hline 11 & $1: 2: 1$ & $90^{\circ} \mathrm{C}$ & $3 \mathrm{~h}$ \\
\hline 12 & $1: 2: 1$ & $90^{\circ} \mathrm{C}$ & $4 \mathrm{~h}$ \\
\hline
\end{tabular}

\subsubsection{Characterization of ZnSe/ZnS Core-Shell QDs.}

Similar to the characterization in Task 1, the synthesized structure will be characterized to check the results. First, high-resolution transmission electron microscopy (HR-TEM) will be used to image and characterize these core-shell structure, especially to determine the thickness of the ZnS shell thickness. Ideally, the shell thickness will be $3 \mathrm{~nm}$ and $5 \mathrm{~nm}$, and the thickness will be complete and uniform for all core-shell structures.

Later on crystalline structure of these quantum dots samples will be determined using powder $\mathrm{x}$ ray diffraction (XRD) techniques to check the structure of the core and shell. To obtain a good result, the peak position of the core structure should not shift and the peak should be sharp for both core and shell structure. In reality, as the crystal structure of both ZnSe and ZnS are Zinc Blende, and the lattice parameter for these two structures are very close to each other, it is acceptable if the peak position for the same facet of these two structures overlap with each other. But the peak intensity of the core-shell structure should be significantly higher than that of the core structure, due to the effect of the shell structure. 
Then photophysical properties of these NCs will be determined by UV-vis spectrometer and laserscanning confocal microscope for absorption properties and Photoluminescence, respectively. And then compare the photophysical properties with that of the ZnSe core QDs in Task 1. For the UVvis absorption spectrum, the intensity of the core-shell structure is not anticipated to be much high than that of the ZnSe core structure and the light absorption range of the core-shell structure is not anticipated to be significantly extended from the ZnSe core structure spectrum. Whereas for the PL spectrum, a good core-shell structure will have much higher intensity than that of the core-only structure.

\subsection{Task 3. Conjugation of ZnSe@ZnS core-shell QDs with human glioma cell line (U251)}

In this task, the synthesized ZnSe@ZnS core-shell QDs from Task 2 will be bioconjugated with human glioma cell line (U251) to detect the effectiveness of these QDs probes, which follows the procedures of Liu. ${ }^{17}$

\subsubsection{Conjugate TGA-Caped ZnSe@ZnS Core-Shell QDs with RGD}

In this sub task, RGD (tripeptide Arg-Gly-Asp, 95\%) solution will be added to the solution of TGA-caped ZnSe@ZnS QDs. React the mixture under stirring at room temperature overnight.

\subsubsection{Culture U251 Cell}

Cells are cultured at $37^{\circ} \mathrm{C}\left(5 \% \mathrm{CO}_{2}\right)$ in Dulbecco's modified Eagle's medium (DMEM), supplemented with $100 \mathrm{mg} / \mathrm{ml}$ penicillin G, 10\% fetal bovine serum (FBS), $1.8 \mathrm{mg} / \mathrm{ml} \mathrm{NaHCO3,}$ and $100 \mathrm{mg} / \mathrm{ml}$ streptomycin sulfate.

\subsubsection{In Vitro Imaging}

Confocal microscopic imaging would be carried out and the cells will be plated onto 14-mm glass coverslips in a 12-well tissue culture plate and are allowed to adhere for $24 \mathrm{~h}$. Afterward, Coreshell QDs-RGD (about $10 \mu \mathrm{g} / \mathrm{ml}$ ) is added to the U251 cells and incubated for $30 \mathrm{~min}$. As a contrast, QDs with no RGD is also added to the U251 cells. 


\section{Chapter 5. Summary}

In this problem report, the synthesis of ZnSe@ZnS core-shell Quantum Dots is proposed for energy and time-saving procedure with trial steps in detail. And the ZnS shell thickness will be tuned by modifying the experiment parameters. As the surfactant utilized to cap these QDs is TGA, which are already hydrophilic surfactants, thus there is no need to conduct surface modification for the bioimaging application test in the next step.

After the synthesis and characterization of the core-shell QDs, once target products are obtained, these core-shell QDs will bioconjugate with human glioma cell line (U251) and conduct in vitro imaging to test the performance of these QDs probes. 


\section{Reference}

1. Fabregat, Isabel. "Dysregulation of apoptosis in hepatocellular carcinoma cells.” World $J$ Gastroenterol 15 (2009): 513-520.

2. Bioimaging. 2017.

http://www.braingut.com/bioimaging.asp

3. Bioimaging 2018. 2017

$<h t t p: / / w w w . b i o i m a g i n g . b i o s t e c . o r g>$

4. Kherlopian, Armen, et al. “A review of imaging techniques for systems biology. “ $B M C$ Systems Biology 2 (2008):74

5. Krishan, Bal and Garg, Meenu. “A literature review on quantum dots.” IJAREEIE 4 (2015): 7857-7862

6. What is a Quantum Dot? 2017

<http://www.dummies.com/education/science/nanotechnology/what-is-a-quantum-dot>

7. Quantum Dots. 2017

$<$ http://www.sigmaaldrich.com/technical-documents/articles/materialsscience/nanomaterials/quantum-dots.html\#ref>

8. Quantum Dots. 2017

<http://www.nanosysinc.com/what-we-do/quantum-dots>

9. Shah, Bhranti, et al. "Labeling of mesenchymal stem cells by bioconjugated quantum dots." Nano Lett 7(2007): 3071-3079.

10. Gao, Xiaohu, et al. "In vivo cancer targeting and imaging with semiconductor quantum dots.”Nat Biotechnol 22 (2004): 969-976.

11. Rahaman, Mohamed and Rahaman Rahaman. "Ceramic Processing and Sintering.” Taylor \& Francis, 2003.

12. Zrazhevskiy, Pavel, Sena Mark and Gao Xiaohu. "Designing multifunctional quantum dots for bioimaging, detection, and drug delivery.” Chem. Soc. Rev. 39 (2010): 4326-4354.

13. Chan, Warren and Shuming Nie, "Quantum dot bioconjugates for ultrasensitive nonisotopic detection.” Science 281 (1998): 2016-2018. 
14. Huang, Jian, et al. "Receptor "hijacking” by malignant glioma cells: A tactic for tumor progression.” Cancer Lett. 267 (2008): 254-261.

15. Chintagumpala, Murali, and Gajjar Amar. "Brain tumors.” Pediatr Clin North Am 62 (2015):167-178.

16. Lacroix, $\mathrm{M}$, et al. "A multivariate analysis of 416 patients with glioblastoma multiforme: Prognosis, extent of resection, and survival,” J. Neurosurg. 95 (2001): 190-198.

17. Liu, Mingxing, et al. "One-pot aqueous synthesization of near-infrared quantum dots for bioimaging and photodynamic therapy of gliomas.” Acta Neurochir Suppl 124 (2017): 303308.

18. Mir, Irshad, et al. “Cadmium-free aqueous synthesis of ZnSe and ZnSe@ZnS core-shell quantum dots and their differential bioanalyte sensing potential.” Mater. Res. Express 3 (2016): 105014.

19. Murray, CB, et al. "Synthesis and characterization of nearly monodisperse CdE (E=S, Se, Te) semiconductor nanocrystallites,” J. Am. Chem. Soc. 115(1993): 8706-8715.

20. Rajh, Tijana, et al. "Synthesis and characterization of surface-modified colloidal CdTe quantum dots," J. Phys. Chem. 97 (1993): 11999- 12003.

21. Ding, C, et al. "Synthesizing quantum dot with uniform grain diameter distribution in water phase comprises preparing molding board agent and cadmium sulfydryl composite precursor, producing water solution of sodium borohydride, and synthesizing quantum dot," East China Normal University, 2010.

22. Jin, Shan, et al. "Review Article-Application of Quantum Dots in Biological Imaging." Journal of Nanomaterials. 2011. 